\title{
CONSTRAINTS ON THE OPERA SUPERLUMINAL NEUTRINOS
}

\author{
PENG-FEI YIN, XIAO-JUN BI, ZHAO-HUAN YU and QIANG YUAN \\ Key Laboratory of Particle Astrophysics, Institute of High Energy Physics, \\ Chinese Academy of Sciences, Beijing 100049, China
}

Received 21 January 2012

Revised 31 January 2012

\begin{abstract}
The OPERA collaboration has recently reported the superluminal neutrinos with velocity of $\left(v_{\nu}-c\right) / c \sim 2.5 \times 10^{-5}$. This result can be explained in the Lorentz invariance violation (LIV) models. The modified dispersion relation of neutrino lead to modified kinematics of the processes the neutrinos involved. For processes that neutrinos in the final states the processes are suppressed. We find to allow the neutrino production process $\pi \rightarrow \mu+\nu_{\mu}$, the neutrino energy should be less than about $5 \mathrm{GeV}$ if taking a large LIV parameter $\sim O\left(10^{-5}\right)$ implied by OPERA data. For the processes that neutrinos in the initial states new processes that are forbidden in the LI scenario may open, such as the $\nu \rightarrow \nu+\gamma$ or $\nu \rightarrow \nu+e^{+}+e^{-}$, which lead neutrinos to lose energy quickly. As the atmospheric neutrino spectrum agrees well with expectation without LIV we find it can constrain the neutrino LIV parameter to $\sim O\left(10^{-12}\right)$, which is 7 orders smaller than the value measured at OPERA. We further propose to study the astrophysical neutrino spectra, which can constrain the neutrino LIV parameter down to $O\left(10^{-15}\right)$.
\end{abstract}

Keywords: OPERA; superluminal neutrino; Lorentz-invariance-violation.

PACS numbers: 13.15.+g, 11.30.Cp, 14.60.St

The OPERA collaboration can measure the velocity of muon neutrinos propagating between the neutrino source beam at the CERN and the OPERA detectors at the underground Gran Sasso Laboratory. Recently, it reported a very surprising result that the neutrinos may be superluminal ${ }^{2}$. The muon neutrinos with average energy $\sim 17 \mathrm{GeV}$ arrived detector earlier than expected from light speed

$$
\delta t=(60.7 \pm 6.9 \text { (stat.) } \pm 7.4 \text { (sys.) }) \mathrm{ns} .
$$

It implied a difference between the muon neutrino velocity and the light speed

$$
\left.\left.\delta_{\nu \gamma}=\left(v_{\nu}-c\right) / c=(2.48 \pm 0.28 \text { (stat. }) \pm 0.30 \text { (sys. }\right)\right) \times 10^{-5} .
$$

This measurement is highly accurate with significance level of $6 \sigma$. The OPERA collaboration has not found the energy dependence of velocity difference $\delta_{\nu \gamma}$. It determined $\delta_{\nu \gamma}=(2.16 \pm 0.76 \pm 0.30) \times 10^{-5}$ for $\langle E\rangle=13.9 \mathrm{GeV}$ and $\delta_{\nu \gamma}=$ $(2.74 \pm 0.74 \pm 0.30) \times 10^{-5}$ for $\langle E\rangle=42.9 \mathrm{GeV}$ respectively. To avoid the debates on 
the long time interval between the source neutrino bunches, the OPERA operation has also done a test performed using a beam with a short-bunch time- structure allowing to measure the neutrino time of flight at the single interaction level. The new measurements are consistent with the previous results ${ }^{2}$.

We expect other neutrino experiments such as MINOS and T2K to perform independent measurements to test the OPERA results in future. Interestingly, some earlier experiments have given compatible results about the velocity of neutrinos with lower accuracy. In 2007 the MINOS experiment at Fermilab reported ${ }^{3}$

$$
\delta_{\nu \gamma}=(5.1 \pm 2.9) \times 10^{-5}
$$

for muon neutrinos with mean energy around $3 \mathrm{GeV}$. It seems to be consistent with that by OPERA. Results from even earlier experiments at Fermilab also obtained a similar value of $\delta_{\nu \gamma}$ at the order of $10^{-54}$. However, the detections of electron antineutrinos from supernova 1987A found the neutrinos arrived on the earth earlier than the relative light only by a time interval of 4 hours. This result can be explained by supernova theory very well. Moreover, neutrino oscillation experiments showed the neutrino velocities are almost flavor independent. Therefore, 1987A results set a very stringent bound on the velocity of neutrinos with energy of $\mathrm{O}(10) \mathrm{MeV}^{5}$

$$
\delta_{\nu \gamma} \lesssim 2 \times 10^{-9}
$$

The superluminal neutrino is obviously in conflict with the special relativity and the standard model, and call for new theory of particle physics. After the OPERA result was reported, many models have been proposed to explain it. Phenomenologically such a superluminal neutrino can be accommodated in a kind of Lorentz invariance violation (LIV) models. In the LIV models the dispersion relation of neutrino is modified as

$$
p^{\mu} p_{\mu}=E^{2}-|\vec{p}|^{2}=m^{2}+f(E,|\vec{p}|) .
$$

The modified neutrino group velocity can be derived as

$$
v_{\nu}=\frac{\partial E}{\partial|\vec{p}|}=\frac{|\vec{p}|}{E}+\frac{1}{2 E} \frac{\partial f}{\partial|\vec{p}|} .
$$

Taking some special $f(E,|\vec{p}|)$, the $v_{\nu}$ larger than $c$ can be obtained.

Studies of effective LIV models which are well motivated by fundamental theory and particle astrophysics detections have a long history. One of the most simple LIV models is so-called 'broken Lorentz invariance violation' model, in which Lorentz symmetry is spontaneous breaking6,7. It preserves linear energy and momentum conservation law and the standard model gauge structure. The ordinary Lorentz transformation related the four-momentum in different reference frames $p_{\mu}^{\prime}=\Lambda_{\mu}{ }^{\nu} p_{\nu}$ is still valid ${ }^{8}$. It should be noted that the value of $p^{\mu} p_{\mu}$ keeps invariant, however the form of $f(E,|\vec{p}|)$ in Eq. (5) is not hold in all the frames. Because the LIV is generated when particles are transformed relative to some unknown background fields in a preferred frame, one can assume a special dispersion relation in such frame, and 
then transform it to other frames. This frame is often chosen to be the 'rest frame of the universe', in which the cosmic microwave background is isotropic ${ }^{6}$. In many cases the earth movement with respect to $\mathrm{CMB}$ can be neglected, the laboratory frame at the earth can be taken as the preferred frame .

Coleman and Glashow (CG) studied detailed phenomenology of such a LIV model where modified energy-momentum relation is assumed $\mathrm{as}^{9}$

$$
E^{2}=m^{2}+|\vec{p}|^{2}+\xi|\vec{p}|^{2} .
$$

Here $\xi \ll 1$ and the Lorentz invariance is violated perturbatively in the context of conventional quantum field theory ${ }^{9}$. Using Eq. (6), for high energy neutrinos we have $\delta_{\nu \gamma}=\xi / 2$.

In one of our recent works ${ }^{1}$ we suggest that the CG model can explain the OPERA superluminal neutrinos and explore the implications of such modified dispersion relation for the processes that neutrinos are involved. The kinematics for those processes including neutrinos is modified and many exotic processes may be induced. This is easily realized from Eq. (7), from which we can define the effective mass of the superluminal neutrinos

$$
m_{e f f}^{2} \equiv m_{\nu}^{2}+\xi|\vec{p}|^{2}=m_{\nu}^{2}+2 \delta_{\nu \gamma}|\vec{p}|^{2} .
$$

We notice from the equation above the superluminal neutrinos become more massive. The mass depends on the momentum at the preferred frame. For those processes the neutrinos are in the final states the processes are suppressed, such as the processes $\pi(K) \rightarrow \mu+\nu_{\mu}, \mu \rightarrow e+\nu_{\mu}+\nu_{e}$ and so on. For the processes that neutrinos are in the initial states some exotic processes that may be forbidden without LIV become possible, such as loop induced process $\nu \rightarrow \nu+\gamma, \nu \rightarrow \nu+e^{+}+e^{-}$by neutral current or the process with charged current $\nu_{\mu} \rightarrow \mu+\nu_{e}+e$. By studying those processes we give stringent constraint on the neutrino LIV parameters, which is much smaller than the value given by OPERA at the $O\left(10^{-5}\right)$.

In reference ${ }^{1}$, we first consider the constraint from $\pi^{+} \rightarrow \mu^{+}+\nu_{\mu}{ }^{\mathrm{a}}$. The pion decay can occur only if neutrino effective mass is not too large according to the threshold condition $m_{e f f, \text { initial }} \geqslant \Sigma m_{\text {eff, final }}$. In the laboratory frame we can get

$$
E_{\nu} \leq\left(m_{\pi}-m_{\mu}\right) \sqrt{1+1 / \xi}
$$

This inequality means that neutrinos generated by pion decay have a maximum energy beyond which the decay process $\pi^{+} \rightarrow \mu^{+}+\nu_{\mu}$ is forbidden.

In Fig. (1) we show the constraints on the neutrino velocity difference $\delta_{\gamma \nu}$ for different neutrino energies with different processes $\pi^{+} \rightarrow \mu^{+}+\nu_{\mu}, K^{+} \rightarrow \mu^{+}+\nu_{\mu}$, $\mu \rightarrow \nu_{\mu}+e+\bar{\nu}_{e}$ and $\tau \rightarrow \nu_{\tau}+e(\mu)+\bar{\nu}_{e(\mu)}$. The region above the line is excluded if we detect the neutrinos from corresponding process. From Fig. (1) we can see that the

\footnotetext{
at should be mentioned that the above threshold conditions actually constrain the difference of the LIV parameters between the parent particle and the daughter particles. In this step, the LIV parameters of pion and muon are assumed to be zero or much smaller than $\xi$.
} 


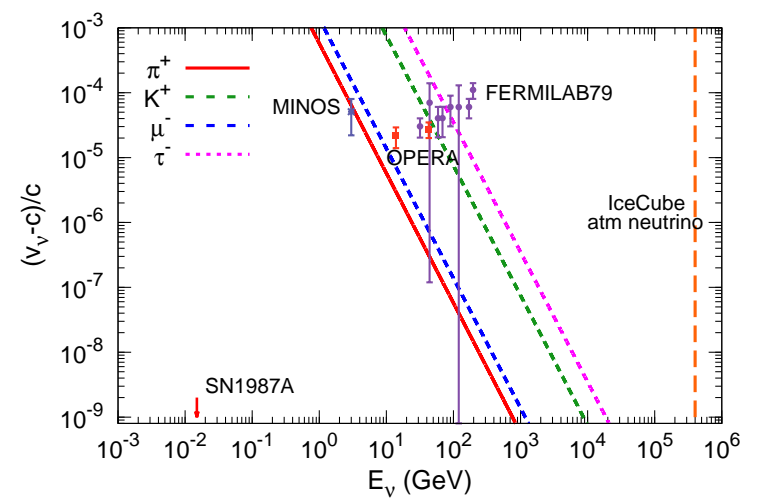

Fig. 1. The neutrino velocity constrained by the neutrino energy, for processes $\pi(K)^{+} \rightarrow \mu^{+} \nu_{\mu}$, $\mu(\tau) \rightarrow \nu_{\mu(\tau)}+e(\mu)+\bar{\nu}_{e(\mu)}$.

process $\pi \rightarrow \mu+\nu_{\mu}$ gives the most stringent constraint. In the OPERA experiment, the neutrinos are just generated by $p p \rightarrow \pi / K \rightarrow \mu+\nu_{\mu}$ at the CERN. It means that the detections of neutrinos with energies as high as $43 \mathrm{GeV}$ by OPERA put a limit of $\delta_{\nu \gamma}<3 \times 10^{-7}$. If we take $\xi \sim O\left(10^{-5}\right)$ as OPERA implied, the neutrino energy can not be larger than several GeV. For the atmospheric neutrinos with energy of $\sim 400 \mathrm{TeV}$ detected by IceCube, they are also produced by pion decay as OPERA neutrinos. In the Fig. (1) we show that the IceCube neutrinos can set very stringent constrain on LIV parameter as $\sim 4 \times 10^{-15}$.

In fact, the original CG model with LIV term $\xi|\vec{p}|^{2}$ induces a constant $\delta v_{\gamma \nu}=$ $\xi / 2$. It can not interpret the OPERA and 1987A results simultaneously. In general, Eq. (7) can be extend as

$$
E^{2}=m^{2}+|\vec{p}|^{2}+\beta|\vec{p}|^{\alpha},
$$

where $\alpha$ is a arbitrary number no less than two and $\beta \sim g^{m} / \Lambda^{n} \ll 1$ is a combination of interaction coefficient $g$ and energy scale $\Lambda$ for some underlying models. If $\alpha=3$, the OPERA and 1987A results can be explained well ${ }^{10}$. In such frameworks, we can get the superluminal neutrino velocity difference and effective mass (neglecting high order terms)

$$
\begin{gathered}
\delta_{\gamma \nu}=\frac{\alpha-1}{2} \beta p^{\alpha-2}, \\
m_{e f f}^{2}=\beta p^{\alpha}=\frac{2 \delta_{\gamma \nu}}{\alpha-1} p^{2} .
\end{gathered}
$$

The constraints on LIV parameters from neutrino energy for $\pi^{+} \rightarrow \mu^{+}+\nu_{\mu}$ are shown in Fig. (2), for different values of $\alpha$. These results show that larger $\alpha$ will give weaker constraints on the parameters.

As we have mentioned above, to give stronger constraint on the $\left|\xi_{\nu}\right| \ll O\left(10^{-5}\right)$ by $\pi^{+} \rightarrow \mu^{+}+\nu_{\mu}$, we need to discuss the bounds on $\xi_{\pi}$ and $\xi_{\mu}$ first $^{11}$ (in the 


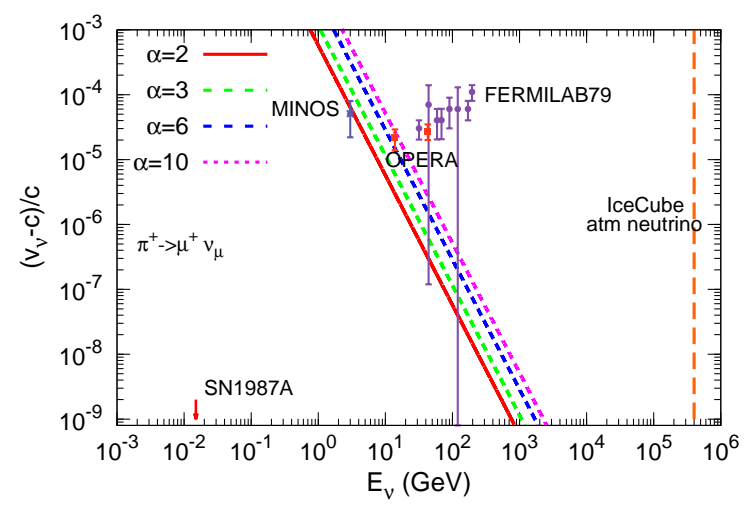

Fig. 2. The neutrino velocity constrained by the neutrino energy for the process $\pi^{+} \rightarrow \mu^{+} \nu_{\mu}$, for different values of $\alpha$.

follows we still focus on CG's model). If the charged pion is also superluminal, it will lose energy quickly by radiative emission $\pi^{+} \rightarrow \pi^{+} \gamma$. The observations of high energy atmospheric neutrinos means pions should not loss energy quickly before they decay to neutrinos. It puts an upper limit of $\xi_{\pi}<10^{-14}$ for pion energy $\sim 2$ $\mathrm{PeV}$. If muon is subluminal, the LIV parameter can be constrained by the photon splitting $\gamma \rightarrow \mu \mu$. As the photons from Crab Nebula with energy of $80 \mathrm{TeV}$ have been detected ${ }^{12}$, we have $\left|\xi_{\mu}\right|<10^{-11}$. Therefore, for the pion decay the bounds on $\left|\xi_{\nu}\right|$ can be set at most to $\sim O\left(10^{-11}\right)$.

We then discuss the processes that neutrinos are in the final states. Two of the processes $\nu \rightarrow \nu+\gamma, \nu_{\mu} \rightarrow \nu_{\mu}+e+e$ can put stronger constraint on the neutrino LIV in principal as only photons and electrons are involved in the process. The

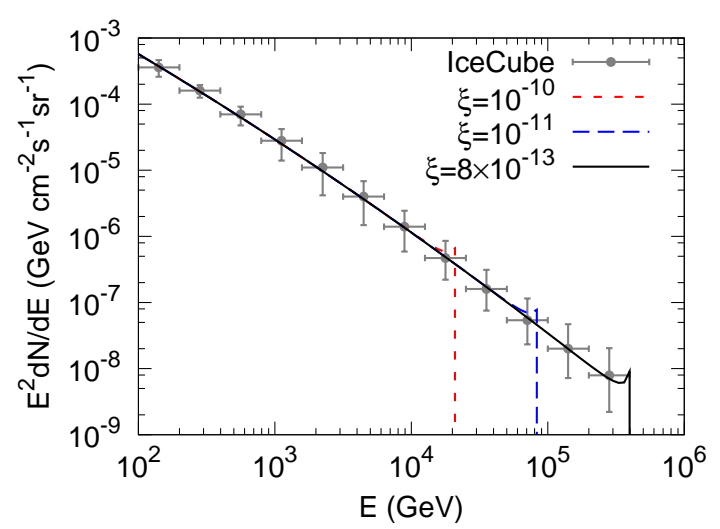

Fig. 3. The expected atmospheric neutrino spectra with LIV parameters $\xi=10^{-10}, 10^{-11}$ and $8 \times 10^{-13}$ respectively. 
processes $e \rightarrow e \gamma$ and $\gamma \rightarrow e e$ give $\left|\xi_{e}\right|<10^{-15}$ by observation of the Crab Nebula gamma rays ${ }^{12}$. As the $\nu \rightarrow \nu+\gamma$ is loop suppressed we discussed the process of $\nu_{\mu} \rightarrow \nu_{\mu}+e+e$ which can in principle set more stringent constraint on the neutrino LIV parameter. This process leads neutrino energy loss rapidly. Using the OPERA baseline of $730 \mathrm{~km}$, Cohen and Glashow claimed the neutrino energies at OPERA detector should be less than $12.5 \mathrm{GeV}$ due to $\nu_{\mu} \rightarrow \nu_{\mu}+e+e^{13}$. The ICARUS experiment also did not observe such energy loss process ${ }^{14}$. Therefore, Cohen and Glashow's work sets a stringent bound on the superluminal neutrino parameter in the LIV model.

We get the energy loss rate by $\nu_{\mu} \rightarrow \nu_{\mu}+e+e$ as

$$
\frac{\mathrm{d} E}{\mathrm{~d} t}=-\langle\Delta E\rangle \Gamma \approx-10^{-3}\left(\frac{E}{\mathrm{TeV}}\right)^{6}\left(\frac{\xi}{10^{-10}}\right)^{3} \mathrm{GeV} \mathrm{s}^{-1},
$$

where $\langle\Delta E\rangle$ and $\Gamma$ are the averaged energy loss of each decay and the decay width (calculation in details in the laboratory system has been given in ${ }^{15}$ ). In our work, we use the method in $^{16}$ to calculate the final neutrino spectra. The detected neutrino spectra can be written as

$$
\phi(E)=\frac{q_{0}\left(E_{0}\right)}{4 \pi d^{2}} \frac{\mathrm{d} E_{0}}{\mathrm{~d} E},
$$

where $q_{0}\left(E_{0}\right)$ is the production spectra per unit time at the source, $d$ is the distance of the source, $E_{0}$ and $E$ are the energies at the source and detector respectively. The energy interval dilation $\mathrm{d} E_{0} / \mathrm{d} E$ is

$$
\frac{\mathrm{d} E_{0}}{\mathrm{~d} E}=\exp \left[\int_{0}^{t=d / c} \frac{\mathrm{d} b\left[E_{0}\left(t^{\prime}\right)\right]}{\mathrm{d} E_{0}\left(t^{\prime}\right)} \mathrm{d} t^{\prime}\right],
$$

where $b(E) \equiv-\mathrm{d} E / \mathrm{d} t$ is the energy loss rate in Eq. (13). Here we only consider the local neutrino sources so we do not need to consider the redshift effect.

For the up-going atmospheric neutrinos observed by IceCube, we take a rough travel distance of $10^{4} \mathrm{~km}$ for the neutrinos with zenith angle $124^{\circ}-180^{\circ}$. In Fig. (3) we show the neutrino spectra with LIV parameters $\xi=10^{-10}, 10^{-11}$, and $8 \times 10^{-13}$ respectively (the measured data by IceCube are also plotted ${ }^{17}$ ). According to the observation of atmospheric neutrinos with $400 \mathrm{TeV}$ at IceCube, we can constrain the LIV parameter of neutrino as $\xi \lesssim 8 \times 10^{-13}$.

Because the astrophysical neutrinos would propagate a very long distance in the universe, their spectra could reveal the information of even smaller LIV effect. Although no high energy astrophysical neutrinos have been detected up to now, we still expect to detect the astrophysical neutrinos from supernova remnants (SNRs) which are well believed the sources of Galactic cosmic rays. Considering much smaller parameter $\xi=10^{-15}$, we show the astrophysical neutrino spectra in the Fig. (4). In the calculation, we consider three distances of the sources 0.3, 1.0 and $3.0 \mathrm{kpc}$, and take source spectrum to be $\propto E^{-2}$. We can see that for sources with distance $\sim \mathrm{kpc}$ the cutoff energy is about several tens to $100 \mathrm{TeV}$ for this small LIV parameter. 


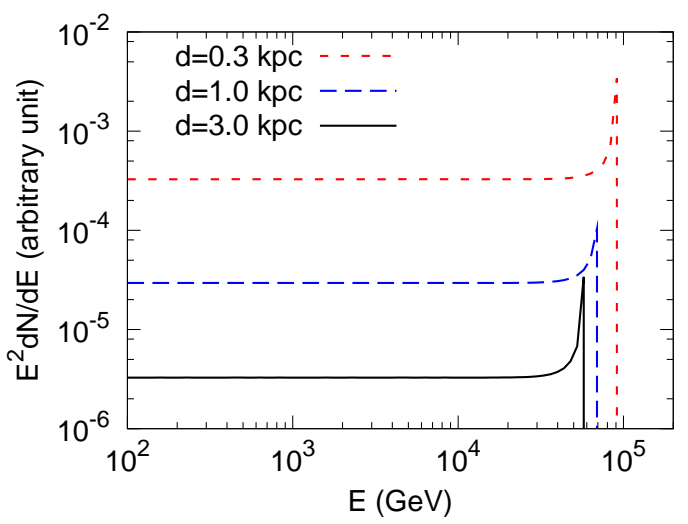

Fig. 4. Expected neutrino spectra with LIV parameter $\xi=10^{-15}$, for sources at distances 0.3 , 1.0 and $3.0 \mathrm{kpc}$ respectively. The source spectrum is assumed as $E^{-2}$.

In this talk, we discuss the implications of the claim of superluminal neutrinos by the OPERA collaboration in the LIV framework. If we adopt $\delta_{\nu \gamma}=2.5 \times 10^{-5}$ as shown by OPERA data, we find the neutrino production process $\pi^{+} \rightarrow \mu^{+}+\nu_{\mu}$ will be forbidden for $E_{\nu}>5 \mathrm{GeV}$. The detection of $\sim 40 \mathrm{GeV}$ and $\sim 200 \mathrm{GeV}$ neutrinos by OPERA and Fermilab can constrain the LIV parameters to less than $3 \times 10^{-7}$ and $2 \times 10^{-8}$ respectively. The neutrino energy loss process $\nu_{\mu} \rightarrow \nu_{\mu}+e+e$ could be used to put stronger limts on the neutrino LIV parameters. This process will result in cutoff of the atmospheric and astrophysical neutrino spectra. The fact no such spectral cutoff of the atmospheric neutrino spectra up to $\sim 400 \mathrm{TeV}$ as measured by IceCube, can constrain neutrino LIV parameter to less than $10^{-12}$. For the astrophysical neutrinos with $\xi \sim 10^{-15}$ and travel distance $\sim \mathrm{kpc}$, the decay process will result in a cutoff at energies about several tens TeV. We hope that such an effect could be tested in future.

\section{Acknowledgments}

This work is supported by the Natural Science Foundation of China under the grant NO. 11075169, and the 973 project under grant No. $2010 \mathrm{CB} 833000$ and by the Chinese Academy of Science under Grant No. KJCX2-EW-W01.

\section{References}

1. X. J. Bi, P. F. Yin, Z. H. Yu and Q. Yuan, Phys. Rev. Lett. 107, 241802 (2011).

2. T. Adam et al. [OPERA Collaboration], arXiv: 1109.4897.

3. P. Adamson at al. [MINOS Collaboration], Phys. Rev. D 76 (2007) 072005.

4. G. R. Kalbfleisch, N. Baggett, E. C. Fowler, J. Alspector, Phys. Rev. Lett. 43, 1361 (1979); J. Alspector et al., Phys. Rev. Lett. 36, 837 (1976).

5. M. J. Longo, Phys. Rev. D 36, 3276 (1987); K. Hirata et al., Phys. Rev. Lett. 58, 1490 (1987); R. M. Bionta et al., Phys. Rev. Lett. 58, 1494 (1987). 
6. S. R. Coleman and S. L. Glashow, Phys. Lett. B 405, 249 (1997).

7. D. Colladay and V. A. Kostelecky, Phys. Rev. D 55, 6760 (1997).

8. J. Alfaro and G. A. Palma, hep-th/0501116.

9. S. R. Coleman and S. L. Glashow, Phys. Rev. D 59, 116008 (1999).

10. G. F. Giudice, S. Sibiryakov and A. Strumia, arXiv:1109.5682 [hep-ph].

11. A. Kostelecky, N. Russell, Rev. Mod. Phys. 83:11 (2011).

12. F. Aharonian et al. [ The HEGRA Collaboration ], Astrophys. J. 614, 897-913 (2004).

13. A. G. Cohen, S. L. Glashow, [arXiv:1109.6562 [hep-ph]].

14. M. Antonello et al. [ICARUS Collaboration], arXiv:1110.3763 [hep-ex].

15. M. Li, D. Liu, J. Meng, T. Wang, L. Zhou, arXiv:1111.3294 [hep-ph]; Y. J. Huo, T. Li and D. V. Nanopoulos, JHEP 1109, 003 (2011).

16. V. Berezinsky, A. Z. Gazizov, S. I. Grigorieva, Phys. Rev. D74, 043005 (2006).

17. R. Abbasi et al. [IceCube Collaboration], Phys. Rev. D83:012001 (2011). 